\title{
A Qualidade Observada em Salas de Berçário
}

\author{
Bárbara Tadeu ${ }^{1}$ \\ Escola Superior de Educação do Instituto Politécnico de Lisboa \\ Cecília Aguiar \\ Instituto Universitário de Lisboa (ISTEC-IUL), CIS-IUL
}

\begin{abstract}
RESUMO - Este estudo teve como objetivo investigar a qualidade de estruturas formais de educação para crianças com menos de 12 meses de idade. Participaram 30 salas de berçário de Setúbal e Palmela, no sul de Portugal. A qualidade global das salas foi avaliada com base na Infant/Toddler Environment Rating Scale - Revised Edition e as práticas de socialização, com base na Caregiver Interaction Scale. Os resultados revelam qualidade global média moderada das salas e elevada qualidade nas práticas de socialização. As associações entre essas medidas de processo e algumas características estruturais das salas sugerem, entre outras implicações, a necessidade de assegurar a formação superior em educação de infância dos profissionais que trabalham nesses contextos de socialização.
\end{abstract}

Palavras-chave: qualidade em creche, interações, berçários

\section{The Quality of Center-Based Child Care and Education for Infants}

\begin{abstract}
This study aimed to investigate the quality of center-based child care and education for infants. Thirty classrooms for infants from Setúbal and Palmela, in the south of Portugal, participated in this study. The overall quality of these classrooms was assessed with the Infant/Toddler Environment Rating Scale - Revised Edition and caregivers' socialization practices were measured with the Caregiver Interaction Scale. Findings suggest that these classrooms present moderate overall quality and high-quality caregiving interaction behaviors. The associations between these process quality measures and selected classroom structural features suggest, among other implications, the need to ensure the presence of professionals with university degrees in early childhood education in these settings.
\end{abstract}

Keywords: child care quality, interactions, infant classrooms

$\mathrm{Na}$ última década, Portugal deparou-se com um crescimento acentuado no número de bebês, menores de um ano, confiados a estruturas educativas de cuidados (creches e amas), visto que, para muitos pais, essas estruturas são a única alternativa para conciliar as exigências da família e do trabalho (United Nations Children's Fund [UNICEF], 2008). De igual forma, o reconhecimento da importância das experiências educacionais durante os primeiros anos de vida (Barros, 2007; Shonkoff \& Philips, 2000) poderá ser outro fator a contribuir para o aumento da procura por esses serviços.

Embora o número de estruturas educativas para a infância tenha vindo a aumentar (Barros, 2007), verifica-se que a taxa de cobertura existente é ainda insuficiente (Conselho Nacional de Educação, 2011). Efetivamente, apesar de o número de creches (i.e., equipamentos socioeducativos destinados ao acolhimento de crianças até aos 3 anos de idade) ter aumentado 53,4\% entre os anos de 1998 e 2009, a taxa de cobertura é de cerca de 33\% (Ministério do Trabalho e da Solidariedade Social, 2009), assegurando um número de vagas insuficiente para satisfazer as necessidades da população.

A literatura sugere que a qualidade das creches pode ter impacto no desenvolvimento das crianças que as frequentam

1 Endereço para correspondência: Instituto Universitário de Lisboa ISCTE, Avenida das Forças Armadas, Lisboa, Portugal. CEP: 1649-026 E-mail: babatadeu@hotmail.com (e.g., Vandell et al., 2010). Assim, deverá atribuir-se a essas instituições um papel importante no desenvolvimento das crianças, tornando-se, assim, essencial avaliar e promover sua qualidade.

Apesar de não serem consensuais, as definições de qualidade dos contextos educativos têm evoluído no sentido de identificar um conjunto de características que podem ser aceitas como indicadores de qualidade geral (Barros, 2007; Pimentel, 2010). A qualidade é, recorrentemente, conceitualizada em duas grandes dimensões: a estrutural e a de processo. A dimensão estrutural refere-se às variáveis relativamente estáveis e passíveis de regulamentação ou fácil mensuração (e.g., razão adulto/criança, tamanho do grupo, educação, formação e experiência dos educadores de infância/auxiliares de ação educativa); a dimensão de processo refere-se a características difíceis de regulamentar ou medir devido à sua natureza qualitativa (e.g., interações adulto/criança e criança/criança; Goelman et al., 2006; Layzer \& Goodson, 2006). Enquanto a dimensão estrutural parece apenas assegurar as condições básicas da qualidade, não sendo suficiente para a determinar (e.g., Cochran, Cochran, \& Torp, 2000), a dimensão de processo associa-se com maior robustez aos resultados desenvolvimentais das crianças (Bryant, Zaslow, \& Burchinal, 2010). Contudo, as duas dimensões não devem ser consideradas isoladamente, uma vez que tendem a interagir, encontrando-se frequentemente associadas (Vandell \& Wolfe, 2000). 
Grande parte da investigação assume que a qualidade reflete o que é propício ao desenvolvimento da criança e, nesse sentido, a National Association for the Education of Young Children (NAEYC, 1997, 2009) define um contexto educativo de elevada qualidade como aquele que proporciona um ambiente seguro e educativo, que promove o desenvolvimento físico, social, emocional, estético, intelectual e da linguagem das crianças, demonstrando sensibilidade em relação às necessidades e preferências das famílias. Assim, para se referir aos programas que levam em consideração essa definição, a NAEYC recorre à designação de práticas desenvolvimentalmente adequadas. A implementação de práticas desenvolvimentalmente adequadas resulta do processo através do qual os profissionais tomam decisões acerca do bem-estar e educação das crianças, com base (a) nos conhecimentos acerca do desenvolvimento e da aprendizagem (adequação à idade); (b) no que é conhecido acerca das forças, interesses e necessidades de cada criança do grupo (adequação às características individuais); e (c) no conhecimento dos contextos sociais e culturais em que as crianças vivem (adequação ao contexto social e cultural). Cada uma destas dimensões é dinâmica e desafiante, exigindo que os profissionais de educação permaneçam aprendizes ao longo das suas vidas profissionais. Dessa forma, a NAEYC responsabiliza os profissionais de educação pela implementação e promoção de padrões de elevada qualidade e profissionalismo nas instituições de educação para a infância, devendo estes refletir conhecimentos atualizados e crenças partilhadas acerca do que constitui educação de elevada qualidade e adequação ao desenvolvimento.

O educador de infância é um elemento chave dentro de uma sala de creche, desempenhando funções fundamentais para assegurar a qualidade do contexto educativo (UNICEF, 2008) e para promover as aprendizagens diárias das crianças (Odom \& Wolery, 2003). Assim, o perfil e funções dos profissionais que trabalham em educação de infância bem como a relação pedagógica estabelecida com as crianças, especialmente em creche, constituem elementos essenciais para a melhoria da qualidade do atendimento às crianças pequenas.

O presente trabalho centra-se em um dos contextos onde se processa o desenvolvimento da criança, assumindo como referência modelos conceituais que consideram o desenvolvimento da criança como o resultado da interação da pessoa e do contexto: o modelo transacional (Sameroff $\&$ Fiese, 2000) e a perspetiva bioecológica (Bronfenbrenner $\&$ Morris, 2006). O presente estudo centra-se, assim, no componente contexto das perspetivas/modelos acima referenciados, procurando contribuir para a avaliação da qualidade das estruturas formais de educação para crianças até os 12 meses.

Em Portugal, os estudos realizados sobre a qualidade em contexto de creche são escassos, incidindo, até ao momento, na área metropolitana do Porto (e.g., Aguiar, Bairrão, \& Barros, 2002; Barros 2007). Tendo em consideração que os resultados dessas avaliações das creches em Portugal têm revelado a existência de uma qualidade pobre ou mínima, é pertinente avaliar mais creches em diferentes regiões do país com a finalidade de se compararem resultados. Por outro lado, visto que os estudos realizados em creche incluíram apenas as faixas etárias dos 1 aos 3 anos de idade, não existem dados relativos às salas de berçário (destinadas a crianças entre $3 \mathrm{e}$ 12 meses de idade), sendo objetivo deste estudo descrever e avaliar a qualidade das salas de berçário em dois municípios do sul de Portugal.

Especificamente, pretende-se (a) descrever a qualidade global das salas de berçário e a qualidade das práticas de socialização dos educadores/auxiliares de ação educativa, (b) estudar a associação entre as práticas de socialização dos profissionais e a qualidade das salas de berçário, (c) estudar as associações entre a qualidade das salas de berçário, as práticas de socialização dos profissionais e variáveis de estrutura relevantes (e.g., razão adulto/criança, tamanho do grupo, educação, formação e experiência dos educadores/ auxiliares de ação educativa).

\section{Método}

\section{Participantes}

Participaram neste estudo 30 salas de berçário pertencentes a 21 instituições dos Municípios de Setúbal ( $n$ $=14)$ e de Palmela $(n=7)$, no sul de Portugal, sendo que destas 13 eram Instituições Particulares de Solidariedade Social (IPSS) e 8 instituições tinham fins lucrativos. Essas instituições foram selecionadas aleatoriamente a partir das listagens disponibilizadas pela Carta Social do Ministério do Trabalho e da Segurança Social. Das 47 creches contactadas, 10 foram consideradas não elegíveis uma vez que a sua ação educativa incidia apenas em crianças a partir dos 12 meses e 16 recusaram participar. Em todos os casos, foi realizado um contato escrito prévio, com posterior confirmação via correio eletrônico. Os educadores de infância e auxiliares de ação educativa participantes receberam informação escrita acerca dos objetivos do estudo e acerca do caráter voluntário da sua participação, tendo sido assegurados o anonimato e a confidencialidade.

Caracterização das salas. Os dados relativos ao tamanho do grupo, à faixa etária das crianças da sala, ao número de adultos na sala, à dimensão das salas e à razão adulto/criança constam na Tabela 1.

Tabela 1. Características estruturais das salas

\begin{tabular}{lrrc}
\hline & M & DP & Min. - Max. \\
\hline Dimensão das salas parque & 20,36 & 4,75 & $14,35-34,98$ \\
Dimensão das salas de sono & 17,96 & 4,49 & $11,13-25,68$ \\
Dimensão total das salas & 38,3 & 8,29 & $26,66-57,00$ \\
N. ${ }^{o}$ de adultos pertencentes à sala & 2,57 & 0,85 & $1-4$ \\
N. $^{\circ}$ de adultos presentes durante a & 2,57 & 0,85 & $1-4$ \\
observação $^{\text {N. }} \begin{array}{l}\text { de crianças que frequentam a } \\
\text { sala }\end{array}$ & 8,17 & 2,29 & $1-13$ \\
$\begin{array}{l}\text { N. }{ }^{o} \text { de crianças presentes durante a } \\
\text { observação }\end{array}$ & 6,90 & 2,41 & $1-10$ \\
Razão adulto/criança & 2,88 & 0,84 & $1-5$ \\
\hline
\end{tabular}


Caracterização das crianças. No que diz respeito às 249 crianças que frequentavam as salas participantes, verificou-se que 123 eram do sexo masculino e 126 , do sexo feminino. A idade das crianças variou entre os 7 e os 29 meses, sendo que a idade mínima presente nas 30 salas observadas variou entre os 7 e os 19 meses $(M=10,43 ; D P=2,57)$ e a idade máxima, entre os 7 e os 29 meses $(M=18,67 ; D P=3,65)$.

Caracterização dos responsáveis oficiais pelas salas e dos profissionais que passavam mais tempo com os bebês. Das 30 salas observadas, $17(56,6 \%$,) estavam sob a responsabilidade de educadores de infância (i.e., profissionais com formação superior em educação de infância) e as 13 restantes $(43,3 \%)$, estavam sob a responsabilidade de auxiliares de ação educativa. Todos os responsáveis oficiais pelas salas eram do sexo feminino.

No que diz respeito ao profissional que passava mais tempo com os bebês, constatou-se que $63,3 \%$ eram auxiliares de ação educativa e $36,7 \%$ eram educadores de infância licenciados (todos do sexo feminino). Entre os auxiliares de ação educativa, um possuía formação superior em educação de infância, no entanto, foi considerado auxiliar de ação educativa devido às funções que constavam no seu contrato de trabalho e ao salário mensal auferido. Dos 18 que não possuíam formação superior, nove possuíam habilitações escolares iguais ou inferiores ao $9 .^{\circ}$ ano de escolaridade e apenas sete concluíram o $12 .^{\circ}$ ano.

A idade dos educadores/auxiliares de ação educativa variou entre 25 e 62 anos $(M=39,73 ; D P=9,29)$ e o tempo de experiência como profissional de educação variou entre 1 e 32 anos $(M=14,47 ; D P=8,88)$. O tempo de experiência na valência de creche variou entre 1 e 24 anos $(M=7,20$; $D P=5,68)$, sendo que o tempo de experiência em salas de berçário variou entre 8 meses e 18 anos $(M=4,34$; $\mathrm{DP}=$ 4,54). O tempo de experiência na instituição variou entre 3 meses e 32 anos $(M=10,79 ; D P=8,20)$. O salário mensal médio auferido foi estimado em 701,19 euros $(D P=281,51)$, variando entre 430,65 e 1700 euros.

\section{Instrumentos}

Escala de Avaliação do Ambiente de Creche. A avaliação da qualidade das salas de creche foi realizada através da aplicação da Escala de Avaliação do Ambiente de Creche - Edição Revista ([ITERS-R], Harms, Cryer, \& Clifford, 2006), uma medida de avaliação da qualidade global de programas para crianças desde o nascimento até aos 30 meses de idade. Foi utilizada a versão portuguesa da escala (Harms, Cryer, \& Clifford, no prelo).

A ITERS-R é constituída por 39 itens organizados em sete áreas ou subescalas conceitualmente definidas: (a) Espaço e Mobiliário, (b) Rotinas de Cuidados Pessoais, (c) Escuta e Conversação, (d) Atividades, (e) Interação, (f) Estrutura do Programa, (g) Pais e Pessoal. A cotação é realizada recorrendo a uma escala de sete pontos $(1=$ inadequado, 3 = mínimo, $5=$ bom e $7=$ excelente) .

Neste estudo, uma vez que as salas observadas eram destinadas a crianças com idades compreendidas entre $3 \mathrm{e}$
12 meses, foram excluídos os itens 17 (Arte), 19 (Blocos), 21 (Brincadeira com areia e água), 23 (Uso de TV, vídeo e computador), 31 (Atividades de grupo) e 32 (Condições para crianças com incapacidades), uma vez que se destinam a salas de atividades para crianças com idades superiores a 12 meses e aos quais, neste caso específico, pode-se atribuir a cotação NA (Não aplicável). Foram igualmente efetuadas análises sem os itens 9 (Fraldas/uso da casa de banho) e 10 (Práticas de saúde) visto apresentarem variância nula. $\mathrm{O}$ coeficiente de alfa de Cronbach para a escala total (31 itens) foi de 0,89 . No que diz respeito às subescalas, os valores do coeficiente alfa de Cronbach oscilaram entre 0,35 na subescala Rotinas de cuidados pessoais e 0,84 na subescala Interação.

Escala de Interação do Prestador de Cuidados. A avaliação das práticas de socialização dos educadores de infância/auxiliares de ação educativa foi realizada através da aplicação da versão portuguesa da Caregiver Interaction Scale (CIS; Arnett, 1989/2011), designada Escala de Interação do Prestador de cuidados (Cadima \& Leal, 2011). Trata-se de uma escala de observação constituída por 26 itens que pretendem avaliar as interações do adulto com as crianças, o seu tom emocional e a abordagem para envolver e disciplinar as crianças. Os 26 itens encontram-se organizados em 4 subescalas: (a) Interação positiva, (b) Punição, (c) Desinvestimento/Não envolvimento e (d) Permissividade (ver Arnett, 1989).

Cada item é apresentado em uma escala de quatro pontos $(1=$ nada, $2=$ um pouco, $3=$ bastante e $4=$ muito $)$, que permite avaliar o grau com que o educador de infância/ auxiliar de ação educativa exibe o comportamento descrito. Na subescala Permissividade, deverá ser efetuada uma inversão na pontuação do item 18 . Para a cotação média global da escala, invertem-se as pontuações dos itens 2, 4, $5,9,10,12,13,15,17,20,21,22,23,24$ e 26 . Uma cotação mais elevada indica interações de maior qualidade (i.e., mais positivas e adequadas).

O coeficiente alfa de Cronbach da CIS foi de 0,97, considerando 25 itens, uma vez que o item 15 foi excluído por não apresentar variabilidade. O coeficiente alfa de Cronbach foi de 0,71 na subescala Permissividade (sem o item 15), 0,88 na subescala Desinvestimento/Não envolvimento e 0,95 nas subescalas Interação Positiva e Punição. Esses indicadores de consistência interna são semelhantes aos resultados relatados no estudo da European Child Care and Education [ECCE] Study Team (1997).

Grade de registro de características estruturais. A grade de registro das características estruturais das instituições e das salas de berçário participantes foi adaptada a partir daquela elaborada por Barros (no prelo). Foram recolhidas informações relativas às características estruturais das instituições e das salas de bebês: tipo de instituição (IPSS ou lucrativa), tamanho do grupo, faixa etária das crianças, número de adultos na sala, dimensão da sala, razão adulto/ criança, categoria profissional do responsável pelas salas, bem como dados relativos ao adulto que passa mais tempo com as crianças (e.g., idade, habilitações acadêmicas, experiência e salário mensal). 


\section{Procedimento}

Treino e acordo interobservadores. Previamente à observação das salas participantes, os observadores participaram em cursos de formação sobre as duas medidas de observação. O treino de aplicação das medidas consistiu na observação de duas salas de berçário que não participaram no estudo, seguida de discussão entre os dois observadores, ambos com formação superior em educação de infância. $\mathrm{O}$ acordo interobservadores foi verificado em 7 das 30 salas ( $23,3 \%$ da amostra). Registrou-se uma percentagem média de acordo exato de $93,07 \%$ nos itens da ITERS-R e de 96,06 nos itens da CIS.

Coleta de dados. Conforme sugerido pelos autores da ITERS-R (Harms et al., 2006), o observador responsável pela coleta dos dados permaneceu em cada uma das salas com o grupo durante 3 horas. A aplicação da CIS ocorreu no decurso do mesmo período de observação. Foram observadas, em todas as salas, as principais rotinas e atividades, desde a chegada das crianças até a ida para a sesta, após o almoço. Todas as salas foram observadas no período da manhã, por ser, geralmente, o período de maior atividade nas creches. Após a observação de cada sala, foi efetuada uma entrevista com o responsável de sala, no sentido de se preencherem os indicadores da escala de difícil observação. No decorrer dessas entrevistas foram, ainda, recolhidas informações relativas às características estruturais das instituições e das salas.

Análise dos dados. O primeiro conjunto de procedimentos incluiu análises exploratórias no sentido de determinar a fidelidade dos dados. Em seguida, foram efetuadas (a) análises descritivas relativas à qualidade das salas de berçário, às práticas de socialização dos educadores de infância/ auxiliares de ação educativa e às características estruturais das instituições e salas e (b) análises inferenciais no sentido de determinar diferenças entre grupos. Calcularam-se, igualmente, coeficientes de correlação através dos quais se procurou descrever as associações simples existentes entre as variáveis em estudo. A análise das distribuições foi realizada através do teste de Kolmogorov-Smirnov e do teste de Shapiro-Wilk. Dada a violação do pressuposto de normalidade nas principais variáveis, foram utilizados testes não paramétricos. O significado prático dos resultados foi interpretado com base nas convenções propostas por Cohen (1992), respeitando o nível de significância de 0,05.

\section{Resultados}

\section{Qualidade das Salas e das Práticas de Socialização}

Os resultados médios obtidos para a qualidade das salas de berçário variam entre 2,39 e 5,68 $(M=4,31 ; D P=0,78)$, indicando uma qualidade média moderada. Duas salas $(6,7 \%)$ apresentam valores médios inferiores a 3 , indicando uma qualidade inadequada e 4 salas $(13,3 \%)$ apresentam valores médios superiores a 5, sugerindo uma boa qualidade. No que se refere às subescalas, as médias variam entre 2,49 na subescala Rotinas e cuidados pessoais e 6,27 na subescala
Interação (ver Tabela 2). Convém salientar que, embora os itens 9 (Fraldas/uso da casa de banho) e 10 (Práticas de saúde) não apresentem variabilidade, são os que apresentam resultados inferiores. Os itens 24 (Interação entre pares) e 25 (Interação pessoal-criança) são os que apresentam resultados médios superiores.

Tabela 2. Médias, desvios-padrão, mínimos e máximos obtidos nas subescalas e nos itens da ITERS-R

\begin{tabular}{|c|c|c|c|}
\hline & $M$ & $D P$ & Mín. - Máx. \\
\hline Subescala Espaço e mobiliário & 4,82 & 1,14 & $2,80-6,80$ \\
\hline 1. Espaço interior & 6,37 & 1,29 & $3-7$ \\
\hline $\begin{array}{l}\text { 2. Mobiliário para cuidados de rotina e } \\
\text { brincadeiras }\end{array}$ & 5,03 & 1,90 & $1-7$ \\
\hline 3. Condições para descanso e conforto & 5,20 & 1,71 & $2-7$ \\
\hline 4. Arranjo da sala & 4,00 & 2,65 & $1-7$ \\
\hline 5. Exposição de material para as crianças & 3,50 & 1,25 & $1-7$ \\
\hline Subescala Rotinas e cuidados pessoais & 2,49 & 0,53 & $1,00-3,50$ \\
\hline 6. Chegada/saída & 5,47 & 1,27 & $1-7$ \\
\hline 7. Refeições/merendas & 1,23 & 1,10 & $1-7$ \\
\hline 8. Sono & 1,53 & 0,50 & $1-2$ \\
\hline 9. Fraldas/uso da casa de banho & 1,00 & 0,00 & 1 \\
\hline 10. Práticas de saúde & 1,00 & 0,00 & 1 \\
\hline 11. Práticas de segurança & 4,70 & 2,03 & $1-7$ \\
\hline Subescala Escuta e conversação & 4,65 & 1,39 & $1,00-6,67$ \\
\hline $\begin{array}{l}\text { 12. Ajudar as crianças a compreender a } \\
\text { linguagem }\end{array}$ & 6,27 & 1,68 & $1-7$ \\
\hline 13. Ajudar as crianças a utilizar a linguagem & 6,07 & 1,98 & $1-7$ \\
\hline 14. Utilizar o livro & 1,63 & 1,45 & $1-6$ \\
\hline Subescala Atividades & 2,80 & 0,76 & $1,67-5,67$ \\
\hline 15. Motricidade fina & 4,30 & 1,51 & $3-7$ \\
\hline 16. Jogo físico ativo & 3,03 & 1,90 & $1-7$ \\
\hline 18. Música e movimento & 3,80 & 1,27 & $2-7$ \\
\hline 20. Jogo dramático & 2,90 & 0,96 & $1-6$ \\
\hline 22. Natureza/ciência & 1,77 & 1,40 & $1-6$ \\
\hline 24. Promover a aceitação da diversidade & 1,03 & 0,18 & $1-2$ \\
\hline Subescala Interação & 6,27 & 1,45 & $2,00-7,00$ \\
\hline 25. Supervisão da brincadeira e da aprendizagem & 6,00 & 2,27 & $1-7$ \\
\hline 26. Interação entre pares & 6,53 & 1,47 & $1-7$ \\
\hline 27. Interação pessoal-criança & 6,53 & 1,35 & $2-7$ \\
\hline 28. Disciplina & 6,03 & 1,81 & $1-7$ \\
\hline Subescala Estrutura do programa & 3,80 & 1,63 & $1,50-7,00$ \\
\hline 29. Horário & 4,57 & 1,63 & $2-7$ \\
\hline 30. Jogo livre & 3,03 & 1,97 & $1-7$ \\
\hline Subescala Pais e pessoal & 3,80 & 1,63 & $1,50-7,00$ \\
\hline 33. Condições para pais & 5,37 & 0,76 & $4-7$ \\
\hline $\begin{array}{l}\text { 34. Condições para necessidades individuais do } \\
\text { pessoal }\end{array}$ & 4,87 & 1,65 & $1-7$ \\
\hline $\begin{array}{l}\text { 35. Condições para necessidades profissionais } \\
\text { do pessoal }\end{array}$ & 5,60 & 1,94 & $1-7$ \\
\hline 36. Interação e cooperação entre o pessoal & 5,44 & 1,82 & $1-7$ \\
\hline 37. Continuidade do pessoal & 5,50 & 1,13 & $1-7$ \\
\hline 38. Supervisão e avaliação do pessoal & 3,00 & 1,89 & $1-7$ \\
\hline $\begin{array}{l}\text { 39. Oportunidades para o desenvolvimento } \\
\text { profissional }\end{array}$ & 3,67 & 1,62 & $1-7$ \\
\hline
\end{tabular}


Os resultados médios obtidos na CIS variam entre 2,56 e 4,00 $(M=3,74 ; D P=0,40)$. Relativamente às subescalas, as médias variam entre 1,12 na subescala Punição e 3,64 na subescala Interação positiva (ver Tabela 3). Os itens 6 (Parece gostar das crianças) e 16 (Fala com as crianças de forma a que elas entendam) são os que apresentam resultados médios superiores e os itens 13 (Passa bastante tempo em atividades que não envolvem a interação com as crianças) e 21 (Não se interessa pelas atividades das crianças) são os que apresentam resultados médios inferiores.

Tabela 3. Médias, desvios-padrão, mínimos e máximos obtidos nas subescalas e nos itens da CIS

\begin{tabular}{|c|c|c|c|}
\hline & $M$ & $D P$ & Mín. - Máx \\
\hline Subescala Interação positiva & 3,64 & 0,56 & $2,20-4,00$ \\
\hline 1. Fala carinhosamente com as crianças & 3,80 & 0,48 & $2-4$ \\
\hline $\begin{array}{l}\text { 3. Escuta atentamente quando as crianças the } \\
\text { falam }\end{array}$ & 3,70 & 0,65 & $2-4$ \\
\hline 6. Parece gostar das crianças & 3,87 & 0,34 & $3-4$ \\
\hline $\begin{array}{l}\text { 7. Quando as crianças se portam mal, explica- } \\
\text { lhes a regra... }\end{array}$ & 3,74 & 0,76 & $1-4$ \\
\hline $\begin{array}{l}\text { 8. Encoraja as crianças a fazerem novas } \\
\text { experiências }\end{array}$ & 3,10 & 1,06 & $1-4$ \\
\hline $\begin{array}{l}\text { 11. Mostra-se entusiasmado com as atividades e } \\
\text { esforços das crianças }\end{array}$ & 3,53 & 0,68 & $2-4$ \\
\hline $\begin{array}{l}\text { 14. Vai ao encontro da individualidade de cada } \\
\text { criança }\end{array}$ & 3,57 & 0,72 & $2-4$ \\
\hline $\begin{array}{l}\text { 16. Fala com as crianças de forma a que elas } \\
\text { entendam }\end{array}$ & 3,90 & 0,30 & $3-4$ \\
\hline $\begin{array}{l}\text { 19. Encoraja as crianças a terem } \\
\text { comportamentos sociais positivos... }\end{array}$ & 3,53 & 0,81 & $1-4$ \\
\hline $\begin{array}{l}\text { 25. Quando fala com as crianças, ajoelha-se, } \\
\text { inclina-se ou senta-se ao nível delas... }\end{array}$ & 3,73 & 0,52 & $2-4$ \\
\hline Subescala Punição & 1,12 & 0,35 & $1,00-2,44$ \\
\hline 2. Mostra-se crítico em relação às crianças & 1,10 & 0,30 & $1-2$ \\
\hline 4. Dá grande valor à obediência & 1,13 & 0,43 & $1-3$ \\
\hline $\begin{array}{l}\text { 10. Fala com as crianças de forma hostil ou } \\
\text { irritada }\end{array}$ & 1,10 & 0,30 & $1-2$ \\
\hline 12. Ameaça as crianças de forma a controlá-las & 1,10 & 0,30 & $1-2$ \\
\hline $\begin{array}{l}\text { 17. Castiga as crianças sem lhes dar uma } \\
\text { explicação }\end{array}$ & 1,13 & 0,43 & $1-3$ \\
\hline 20. Facilmente encontra defeitos nas crianças & 1,07 & 0,25 & $1-2$ \\
\hline $\begin{array}{l}\text { 22. Proíbe muitas das coisas que as crianças } \\
\text { querem fazer }\end{array}$ & 1,27 & 0,78 & $1-4$ \\
\hline 24. Espera das crianças autocontrolo... & 1,07 & 0,25 & $1-2$ \\
\hline 26. Mostra-se, desnecessariamente, severo... & 1,13 & 0,43 & $1-3$ \\
\hline Subescala Desinvestimento/Não envolvimento & 1,35 & 0,51 & $1,00-2,75$ \\
\hline 5. Parece distante ou indiferente... & 1,27 & 0,52 & $1-3$ \\
\hline $\begin{array}{l}\text { 13. Passa bastante tempo em atividades que não } \\
\text { envolvem a interação com crianças }\end{array}$ & 1,43 & 0,62 & $1-3$ \\
\hline $\begin{array}{l}\text { 21. Não se interessa pelas atividades das } \\
\text { crianças }\end{array}$ & 1,40 & 0,62 & $1-3$ \\
\hline 23. Não supervisiona as crianças de muito perto & 1,33 & 0,60 & $1-3$ \\
\hline Subescala Permissividade & 1,13 & 0,34 & $1,00-2,50$ \\
\hline 9. Exerce pouco controlo sobre as crianças & 1,13 & 0,34 & $1-2$ \\
\hline $\begin{array}{l}\text { 15. Não repreende as crianças quando se } \\
\text { portam mal }\end{array}$ & 1,00 & 0,00 & 1 \\
\hline 18. Mostra firmeza quando necessário & 1,13 & 0,43 & $1-3$ \\
\hline
\end{tabular}

\section{Associações entre a Qualidade das Salas de Berçário, as Práticas de Socialização e as Características Estruturais}

O cálculo do coeficiente rho de Spearman permitiu apurar uma associação positiva forte, estatisticamente significativa, entre a média da qualidade das salas e a média das práticas de socialização dos educadores de infância/auxiliares de ação educativa $\left(r_{s}=0,58\right)$. Foram igualmente verificadas as associações entre a qualidade das salas e determinadas características de estrutura: tamanho do grupo de crianças, número de adultos da sala, razão adulto/criança, categoria profissional, habilitações acadêmicas, idade, salário mensal auferido e anos de experiência do profissional que passa mais tempo com os bebês. Foi encontrada uma associação positiva moderada, estatisticamente significativa, entre a média da qualidade das salas e o tamanho do grupo $\left(r_{s}=0,37\right)$ e uma associação positiva forte, estatisticamente significativa, entre a qualidade das salas e o número de adultos na sala $(r$ $=0,63)$. Do mesmo modo, foi encontrada uma associação negativa moderada, estatisticamente significativa, entre a qualidade das salas e a categoria profissional do adulto que passa mais tempo com os bebês $\left(r_{s}=-0,46\right)$, bem como uma associação positiva moderada, estatisticamente significativa, entre a qualidade das salas e as habilitações acadêmicas do profissional $\left(r_{s}=0,49\right)$. Relativamente às associações entre a qualidade das salas e a média da razão adulto/criança observada, e a idade e tempo de experiência em educação (em creche e na instituição) do profissional que passa mais tempo com os bebês, não foram encontradas associações estatisticamente significativas. Contudo, foi encontrada uma associação negativa moderada, estatisticamente significativa, entre a qualidade das salas e o tempo de experiência do profissional em salas de berçário $\left(r_{s}=-0,49\right)$. No que diz respeito às associações entre a qualidade das salas e o salário mensal auferido pelo profissional que passa mais tempo com os bebês foi encontrada uma associação positiva forte, estatisticamente significativa $\left(r_{s}=0,57\right)$. Finalmente, foram verificadas as associações entre a qualidade das práticas de socialização dos educadores de infância/auxiliares de ação educativa e as características de estrutura descritas previamente; contudo, não foram encontradas quaisquer associações estatisticamente significativas.

\section{Comparação da Qualidade das Salas e das Práticas de Socialização em Função de Caraterísticas Estruturais}

De acordo com os objetivos de investigação, foram analisados os efeitos do tipo de instituição, da categoria profissional do responsável pela sala dos bebês e da categoria profissional do adulto que passa mais tempo com os bebês na qualidade das salas. De acordo com a informação que consta na Tabela 4, existem diferenças estatisticamente significativas, de elevada magnitude, na qualidade das salas de berçário em função do tipo de instituição, da categoria profissional do adulto responsável pela sala e da categoria profissional do adulto que passa mais tempo com os bebês. Especificamente, a qualidade média das salas era superior em IPSS, em salas cujo responsável é um educador de infância 
Tabela 4. Teste de Mann-Whitney para o efeito do tipo de instituição e da categoria profissional do responsável de sala e do adulto que passa mais tempo com os bebês na qualidade das salas e nas práticas de socialização

\begin{tabular}{|c|c|c|c|c|c|c|c|c|}
\hline & \multicolumn{4}{|c|}{ Qualidade das salas } & \multicolumn{4}{|c|}{ Práticas de socialização } \\
\hline & $M(D P)$ & $U$ & $p$ & $d$ & $M(D P)$ & $U$ & $p$ & $D$ \\
\hline \multicolumn{9}{|l|}{ Tipo de instituição } \\
\hline IPSS $(n=22)$ & $4,46(0,79)$ & \multirow{2}{*}{42,500} & \multirow{2}{*}{0,031} & \multirow{2}{*}{0,79} & $3,74(0,42)$ & \multirow{2}{*}{83,000} & \multirow{2}{*}{0,836} & \multirow{2}{*}{$-0,07$} \\
\hline Lucrativa $(\mathrm{n}=8)$ & $3,91(0,63)$ & & & & $3,77(0,40)$ & & & \\
\hline \multicolumn{9}{|c|}{ Categoria profissional do responsável da sala } \\
\hline Educador de infância $(\mathrm{n}=17)$ & $4,72(0,49)$ & \multirow{2}{*}{32,500} & \multirow{2}{*}{0,001} & \multirow{2}{*}{1,41} & $3,87(0,28)$ & \multirow{2}{*}{60,500} & \multirow{2}{*}{0,035} & \multirow{2}{*}{0,73} \\
\hline Auxiliar de ação educativa $(\mathrm{n}=13)$ & $3,79(0,78)$ & & & & $3,58(0,50)$ & & & \\
\hline \multicolumn{9}{|c|}{ Categoria profissional do adulto que passa mais tempo com os bebês } \\
\hline Educadora de Infância $(\mathrm{n}=11)$ & $4,76(0,52)$ & \multirow{2}{*}{46,000} & \multirow{2}{*}{0,011} & \multirow{2}{*}{1,05} & $3,90(0,15)$ & \multirow{2}{*}{82,000} & \multirow{2}{*}{0,350} & \multirow{2}{*}{0,70} \\
\hline Auxiliar de acão educativa $(\mathrm{n}=19)$ & $4,06(0,80)$ & & & & $3,66(0,48)$ & & & \\
\hline
\end{tabular}

e em salas em que o profissional que passa mais tempo com os bebês é um educador de infância.

Existem, ainda, diferenças estatisticamente significativas, de magnitude moderada, nas práticas de socialização em função da categoria profissional do adulto responsável pela sala. No que diz respeito à categoria do profissional que passa mais tempo com os bebês não foram encontradas diferenças estatisticamente significativas, embora se tenha encontrado um efeito de magnitude moderada. Não se verificaram diferenças nas práticas de socialização dos educadores de infância/auxiliares de ação educativa em função do tipo de instituição.

\section{Discussão}

\section{Qualidade das Salas e das Práticas de Socialização}

De acordo com os resultados obtidos, a qualidade média das salas de berçário nos municípios de Setúbal e Palmela pode ser considerada moderada. Esses níveis médios de qualidade revelam-se superiores aos resultados obtidos em estudos previamente realizados em Portugal (e.g., Aguiar et al., 2002; Barros, 2007) e aos resultados de alguns estudos desenvolvidos na Europa e nos Estados Unidos (ver Barros \& Aguiar, 2010), em contexto de creche. Sendo inesperados, esses resultados levantam um conjunto relevante de questões, que, por sua vez, podem permitir a formulação de novas hipóteses de investigação: (a) Considerando que a última avaliação da qualidade de salas de creche foi conduzida em 2004/2005 (Barros, 2007), será que esses resultados evidenciam uma evolução positiva das características dos contextos de educação e cuidados destinados às crianças mais novas, nos últimos anos? (b) Uma vez que as avaliações prévias da qualidade de salas de creche se realizaram na área metropolitana do Porto, será que esses dados espelham assimetrias regionais na qualidade desses contextos? (c) Considerando que as avaliações anteriores da qualidade das creches foram conduzidas em salas destinadas a crianças entre 12 e 36 meses de idade, será que os dados obtidos refletem níveis de qualidade superiores nas condições proporcionadas a crianças mais novas? (d) Uma vez que os dados relatados neste estudo foram obtidos por uma equipe diferente, com formação distinta, será que esses resultados podem ser atribuídos a efeitos dos observadores?

Apesar das diferenças registadas nos níveis de qualidade relatados, interessa salientar que os itens que apresentam maiores fragilidades (áreas deficitárias) e os itens que expressam maior qualidade (áreas fortes) parecem convergir, em termos relativos, com o perfil de qualidade descrito em estudos prévios. Assim, e à semelhança de outros estudos (e.g., Aguiar et al., 2002; Barros, 2007; Barros, Pinto, \& Leal, 2012; Goelman et al., 2006; Pessanha, Aguiar, \& Bairrão, 2007), as dimensões de qualidade identificadas como inadequadas estão relacionadas as subescalas Rotinas de cuidados pessoais e Atividades, incluindo (a) as práticas de higiene e saúde; (b) os procedimentos básicos de higiene relativos às refeições e à preparação dos alimentos; (c) a supervisão do sono das crianças; (d) a distância entre os berços; (e) a oferta e estado de conservação de livros; (f) existência de imagens, livros e brinquedos que representam a natureza e as ciências de forma realista; (g) a existência de materiais que evidenciem diversidade étnica ou cultural; e (h) a frequência de atividades/idas ao exterior.

Em contrapartida, na maioria das salas observadas, verificou-se a existência de espaços e mobiliários de boa qualidade, indicando algum investimento nas características estruturais dessas instituições. Foram, igualmente, verificadas boas práticas por parte dos educadores de infância/auxiliares de ação educativa relativamente ao uso da linguagem e em todos os itens que constituem a subescala Interação, sendo esta a subescala que apresenta resultados médios mais elevados. Assim, os resultados obtidos, quer nos itens relacionados com a linguagem, quer nos itens da subescala Interação, em termos relativos, vão no sentido dos resultados obtidos por Aguiar et al. (2002), Barros (2007) e Barros et al. (2012).

Tendo em conta a média obtida na CIS, pode considerar-se que as práticas de socialização e de interação dos educadores de infância/auxiliares de ação educativa são, na sua maioria, de elevada qualidade. Recorrendo ao mesmo instrumento, o ECCE Study Group (1997) relatou, igualmente, níveis elevados de qualidade, com diferenças mínimas entre os 
países participantes (i.e., Alemanha, Áustria, Espanha, EUA e Portugal). Note-se, contudo, que resultados recentemente divulgados sugerem limitações em termos do potencial dessa escala para discriminar educadores de infância/auxiliares de ação educativa com práticas moderada e altamente positivas nas suas interações com as crianças (Colwell, Gordon, Fujimoto, Kaestner, \& Korenman, 2013).

\section{Relações entre a Qualidade das Salas de Berçário, as Práticas de Socialização e as Características Estruturais}

De acordo com os resultados obtidos, a qualidade das salas está fortemente correlacionada com as práticas de socialização dos educadores de infância/auxiliares de ação educativa. Essa associação pode justificar-se pelo fato de a CIS avaliar um dos aspetos processuais da qualidade (i.e., as interações adulto-criança) que se encontra contemplado na escala ITERS-R. Note-se, contudo, que apesar de ser considerada uma medida de qualidade de processo (Vandell \& Wolfe 2000), a ITERS-R avalia a qualidade geral das salas incluindo, concomitantemente, aspectos estruturais e aspectos processuais. Assim, essa associação pode refletir a relação entre dimensões de estrutura e de processo nas experiências proporcionadas às crianças.

A razão adulto/criança tem sido considerada a medida de qualidade estrutural que melhor prediz a qualidade das interações adulto/criança (e.g., Whitebook, Howes, \& Phillips, 1989). No presente estudo, a média da razão adulto/ criança foi 1:3, correspondente à proporção recomendada por vários autores (e.g., Bredekamp, 1987; Howes, Phillips, \& Whitebook, 1992), segundo os quais, em salas de creche com crianças muito pequenas, o número de crianças deve ser reduzido, de forma a proporcionar interações diádicas educador-criança, possibilitando um conhecimento profundo das características de cada criança.

No presente estudo, em conformidade com vários outros (e.g., Barros, 2007; Phillipsen, Burchinal, Howes, \& Cryer, 1997), verificou-se uma associação positiva entre o número de adultos e a qualidade da sala. Contudo, não foi encontrada uma associação entre a qualidade média das salas e a razão adulto/criança observada. Relativamente ao tamanho do grupo, a qualidade média das salas parece aumentar quando as salas são frequentadas por mais crianças. Seria de esperar que, em uma sala com menos crianças, fossem fornecidos serviços de qualidade mais elevada. No entanto, tal não acontece, o que pode estar relacionado com o fato de a qualidade média das salas aumentar à medida que aumenta o número de adultos por sala. Goelman et al. (2006) constataram que a qualidade média das salas aumentava por cada adulto adicional na sala e realçaram que pode existir uma grande diferença entre uma sala com uma razão adulto/ criança de 1:4 e uma sala com igual razão mas que tenha dois adultos para oito crianças, pois o fato de existirem dois adultos parece oferecer vantagens no que tange à supervisão das crianças, fornecendo, igualmente, a possibilidade de os adultos partilharem e discutirem planejamentos, experiências e procedimentos profissionais.
No que concerne ao responsável oficial pelas salas, verificou-se que a qualidade das salas aumenta quando o profissional é educador de infância e diminui quando este profissional é auxiliar de ação educativa. Também no que concerne ao profissional que passa mais tempo com os bebês se verificou que a média da qualidade das salas é superior quando estes são educadores de infância. Similarmente, as práticas de socialização apresentam resultados superiores quando o responsável oficial pela sala é um educador de infância. Esses resultados são convergentes com os resultados relatados por Pessanha et al. (2007) e Barros (2007), contribuindo para a acumulação de evidências empíricas que fundamentam a necessidade de assegurar a formação superior em educação de infância dos profissionais que trabalham em creche.

Tal como ocorreu no estudo de Barros (2007), não foram encontradas associações entre a qualidade das salas e a idade e o tempo de experiência em educação (em creche e na instituição) do profissional que passa mais tempo com os bebês. No entanto, foi encontrada uma associação negativa entre a qualidade média das salas e o tempo de experiência do profissional em salas de berçário, emergindo um efeito que merece replicação, uma vez que remete para a eventual necessidade de considerar, de modo muito particular, as exigências do trabalho desenvolvido nessas salas e as necessidades de apoio, formação em serviço e supervisão dos profissionais. Uma vez que vários estudos internacionais apontam para o fato de educadores mais velhos e mais experientes prestarem educação e cuidados em salas com melhor qualidade (e.g., van Ijzendoorn, Tavecchio, Stams, Verhoeven, \& Reiling, 1998), os resultados obtidos sobre o tempo de experiência em berçário vão contra as nossas expectativas. São, contudo, parcialmente convergentes com os resultados relatados por Pessanha et al. (2007), segundo os quais existe uma relação negativa entre a idade dos educadores e a qualidade da sala. Segundo Pessanha et al., o trabalho nas salas de creche é exaustivo, os salários são bastante modestos e a maioria dos cuidadores não têm oportunidade de serem promovidos dentro ou fora da instituição, o que significa que não há incentivos externos para proporcionar uma boa qualidade de serviços. Assim, de acordo com esses autores, os educadores de infância/ auxiliares de ação educativa mais jovens podem (a) conseguir lidar melhor com as condições desfavoráveis de trabalho e as limitadas perspectivas de carreira ou (b) estar mais bem preparados para a prática, dada a sua formação mais recente e atualizada.

Relativamente ao salário, nesta amostra, um profissional auferia um salário mensal inferior ao salário mínimo nacional e, em média, os salários eram relativamente baixos, o que sugere uma desvalorização social da educação e cuidados de crianças com menos de 12 meses de idade. Paralelamente, encontrou-se uma associação positiva forte entre o salário mensal do educador de infância/auxiliar de ação educativa e a qualidade média das salas (mas não entre o salário e as práticas de socialização). Vários estudos (e.g., Goelman et al., 2006; Phillipsen et al., 1997) têm encontrado associações positivas entre o salário auferido pelo educador e a qualidade global de processo das salas de creche e de jardim-deinfância, sugerindo que o recrutamento de educadores mais 
qualificados e competentes, garantindo-lhes um salário mais atrativo, é uma medida conducente à qualidade.

Em Portugal, também Pessanha et al. (2007) revelaram uma associação positiva entre o salário do responsável pela sala de creche e a qualidade média das salas. Naturalmente, essa relação positiva pode ser explicada, pelo menos parcialmente, pela categoria profissional dos profissionais, uma vez que, geralmente, os educadores de infância auferem salários mais elevados do que os auxiliares de ação educativa. Contudo, outras explicações são plausíveis, nomeadamente explicações relacionadas com processos motivacionais e com a valorização social e remuneratória dos profissionais por parte de instituições comprometidas com a educação de elevada qualidade. A futura confirmação de que o salário dos educadores de infância/auxiliares de ação educativa constitui um fator facilitador da qualidade de processo afigura-se relevante uma vez que se trata de uma variável que pode ser alvo de regulamentação.

Finalmente, e contrapondo os resultados de Aguiar e colaboradores (2002), segundo os quais, a qualidade média das salas de instituições com fins lucrativos era superior à qualidade média das salas das IPSS, no presente estudo, verificou-se que a qualidade média das salas de IPSS era superior à qualidade média das salas de instituições com fins lucrativos. Esses resultados são convergentes com estudos internacionais prévios (e.g., Cost, Quality, \& Child Outcomes Study Team 1995) e, podem dever-se, entre outros fatores, ao fato de serem concedidos mais apoios estatais às IPSS. Note-se que não se verificaram diferenças nas práticas de socialização em função do tipo de instituição.

\section{Limitações}

Os resultados deste estudo devem ser interpretados à luz de um conjunto de limitações. Assim, a amostra de salas de berçário utilizada neste estudo tem um tamanho reduzido e encontra-se circunscrita aos municípios de Setúbal e Palmela, não sendo equilibrada no que se refere aos dois tipos de instituição incluídos, uma vez que o número de creches de IPSS é superior ao número de instituições lucrativas. Assim, os resultados deste estudo não podem ser generalizados a outras áreas do país e mesmo ao distrito de Setúbal.

O procedimento de análise de dados não levou em consideração o fato de as 30 salas observadas pertencerem a 21 instituições, não controlando, portanto, o fator instituição. De igual forma, pretendia-se que este estudo incluísse apenas salas de berçário com crianças de idades compreendidas entre os 3 e os 12 meses. Contudo, no decurso do processo de coleta de dados, verificou-se que, em cinco das 30 salas da amostra, a idade mínima das crianças ultrapassava os 12 meses e que, apenas em uma sala, a idade máxima das crianças não ultrapassava os 12 meses.

Do ponto de vista conceitual, o presente trabalho centrouse em um dos contextos onde se processa o desenvolvimento da criança, assumindo como referência o modelo transacional (Sameroff \& Fiese, 2000) e a perspetiva bioecológica (Bronfenbrenner \& Morris, 2006). No entanto, não foram estudadas as quatro componentes do modelo pessoa- processo-contexto-tempo ou os processos de interação recíproca das crianças e das características das creches, nem foi estudado o impacto da qualidade dos contextos no desenvolvimento das crianças, pelo que se sugere que futuras investigações contemplem esses aspectos.

\section{Implicações}

Os resultados obtidos demonstram a necessidade de melhorar certos aspectos da qualidade dos cuidados prestados às crianças mais novas, nomeadamente no que diz respeito à higiene e saúde e às atividades proporcionadas às crianças. Uma vez que os resultados do estudo indicam que a subescala Atividades corresponde a uma das áreas deficitárias e visto que a valência de creche, a nível nacional, possui escassos documentos orientadores que contemplem a intencionalidade da prática educativa para as crianças dos 0 aos 3 anos, recomenda-se a criação de um referencial orientador da prática pedagógica que promova "a agência da criança e a sua participação, celebrem o jogo e a brincadeira, integrem educação e cuidados, integrem a escola e a família" (Formosinho, 2010, p. 80).

De acordo com os resultados deste estudo, justifica-se o investimento na formação inicial e contínua dos educadores de infância, na medida em que a qualidade das salas dirigidas por educadores de infância e em que este é o profissional que passa mais tempo com os bebês apresenta resultados superiores. No entanto, parece-nos igualmente importante incentivar a melhoria de mecanismos de supervisão pedagógica (Formosinho, 2010) e de avaliação das práticas. Nesse sentido, o Ministério que tutela a creche deverá mobilizar esforços no sentido de as visitas de inspeção não se restringirem a questões formais e à verificação de condições estruturais, mas abrangerem a supervisão e avaliação dos currículos e das práticas.

\section{Referências}

Aguiar, C., Bairrão, J., \& Barros, S. (2002). Contributos para o estudo da qualidade em contexto de creche na área metropolitana do Porto. Infância e Educação: Investigação e Práticas, 5, 7-28.

Arnett, J. (1989). Caregivers in day-care centers: Does training matter? Journal of Applied Developmental Psychology, 10, 541-552.

Barros, S. (2007). Qualidade em contexto de creche: Ideias e práticas (Tese de Doutoramento). Universidade do Porto, Portugal. Recuperado de http://repositorio-aberto.up.pt/ bitstream/10216/19498/2/87542.pdf

Barros, S. (no prelo). Grelha de registo de características estruturais das instituições e das salas de berçário. Universidade do Porto, Portugal

Barros, S., \& Aguiar, C. (2010). Assessing the quality of Portuguese child care programs for toddlers. Early Childhood Research Quarterly, 25, 527-535. doi:10.1016/j.ecresq.2009.12.003 
Barros, S., Pinto, A. I., \& Leal, T. (2012). Qualidade em contexto de creche e envolvimento de grupo. In N. Ramos, E. Mendes, A. I. Silva, \& J. Porfírio (Orgs.), Família, educação e desenvolvimento no séc. XXI: Olhares interdisciplinares (pp. 91-97). Escola Superior de Educação, Instituto Politécnico de Portalegre, Portugal.

Bredekamp, S. (1987). Developmentally appropriate practices in early childhood programs serving children from birth through age 8: Revised Edition. Washington, DC: National Association for the Education of Young Children.

Bronfenbrenner, U., \& Morris, P. A. (2006). The bioecological model of human development. In W. Damon \& R. M. Lerner (Eds.), Handbook of child psychology, Vol. 1: Theoretical models of human development (6th ed., pp. $793-828)$. New York, NY: Wiley.

Bryant, D. M., Zaslow, M., \& Burchinal, M. (2010). Issues in measuring quality programs. In P. Wesley \& V Buysse (Eds.), The quest for quality: Promising innovations for early childhood programs (pp. 47-68). Baltimore, MD: Brookes.

Cadima, J., \& Leal, T. (2011). Escala de Interação do Prestador de Cuidados: Manual de aplicação. Universidade do Porto, Portugal.

Cochran E., Cochran, M., \& Torp, N. (2000). Choosing highquality child care. Recuperado de http://ecommons.cornell. edu/bitstream/1813/3906/2/Choosing\%20High-Quality\%20 Child\%20Care.pdf

Cohen, J. (1992). Quantitative methods in Psychology: A power primer. Psychological Bulletin, 112, 155-159.

Colwell, N., Gordon, R. A., Fujimoto, K., Kaestner, R., Korenman, S. (2013). New evidence on the validity of the Arnett Caregiver Interaction Scale: Results from the Early Childhood Longitudinal Study-Birth Cohort. Early Childhood Research Quarterly, 28(2), 218-23. http://dx.doi.org/10.1016/j. ecresq.2012.12.004

Conselho Nacional de Educação. (2011). Recomendação n. ${ }^{\circ}$ 3/2011: A educação dos 0 aos 3 anos. Diário da República, 2. ${ }^{a}$ Série - N. ${ }^{\circ}$ 79, de 21 de Abril de 2011.

Cost, Quality \& Child Outcomes Study Team. (1995). Cost, quality, and child outcomes in child care centers: Public report $\left(2^{\mathrm{a}} \mathrm{ed}\right.$.). University of Colorado at Denver.

European Child Care and Education Study Group. (1997). European child care and education study: Cross national analyses of the quality and effects of early childhood programmes on children's development. Final report for work package \#1 submitted to European Union DG XII: Science, Research and Development RTP Action: Targeted Socio-Economic Research. Manuscrito não publicado.

Formosinho, J. (2010). Educação das crianças até aos três anos: Algumas lições da investigação. In Conselho Nacional de Educação (Ed.), A educação das crianças dos 0 aos 3 anos: Estudos e seminários (pp. 61-91). Lisboa, Portugal: Conselho Nacional de Educação.

Goelman, H., Forer, B., Kershaw, P., Doherty, G., Lero, D., \& LaGrange, A. (2006). Towards a predictive model of quality in Canadian child care centers. Early Childhood Research Quarterly, 21, 280-295.

Harms, T., Cryer, D., \& Clifford, R. M. (2006). Infant/Toddler Environment Rating Scale: Revised Edition. New York, NY: Teachers College Press.
Harms, T., Cryer, D., \& Clifford, R. M. (no prelo). Escala de Avaliação do Ambiente de Creche: Edição Revista (Infant/ Toddler Environment Rating Scale: Revised Edition; S. Barros, A. I. Pinto, C. Peixoto, \& M. Pessanha, Trans.). Porto, Portugal: Universidade do Porto .

Howes, C., Phillips, D. A., \& Whitebook, M. (1992). Thresholds of quality: Implications for the social development of children in center-based child care. Child Development, 63, 449-460.

Layzer, J. I., \& Goodson, B. D. (2006). The "quality" of early care and education settings: Definitional and measurement issues. Evaluation Review, 30(5), 556-576. doi: $10.1177 / 0193841$ X06291524

Ministério do Trabalho e da Solidariedade Social. (2009). Carta Social, rede de serviços e equipamentos: Relatório 2009. Recuperado de http://www.cartasocial.pt/relatorios.php\#.

National Association for the Education of Young Children. (1997). Developmentally appropriate practice in early childhood programs serving children from birth through age 8: A position statement of the National Association for the Education of the Young Children. Washington, DC: NAEYC.

National Association for the Education of Young Children. (2009). Position statement: NAEYC Developmentally Appropriate Practice in Early Childhood Programs Serving Children from Birth through Age 8. Recuperado de https://www.naeyc.org/ files/naeyc/file/positions/position\%20statement\%20Web.pdf

Odom, S., \& Wolery, M. (2003). A unified theory of practice in early intervention/early childhood special education: Evidence based practices. Journal of Special Education, 37(3), 164-174.

Pessanha, M., Aguiar, C., \& Bairrão, J. (2007). Influence of structural features on Portuguese toddler child care quality. Early Childhood Research Quarterly, 22, 204-214.

Phillipsen, L. C., Burchinal, M. R., Howes, C., \& Cryer, D. (1997). The prediction of process quality from structural features of child care. Early Childhood Research Quarterly, 12(3), 281-303.

Pimentel, J. S. (2010). Atendimento e intervenção precoce a crianças em risco dos 0 aos 3 anos: Em busca da qualidade. In Conselho Nacional de Educação (Ed.), A educação das crianças dos 0 aos 3 anos: Estudos e seminários (pp. 121-128). Lisboa, Portugal: Conselho Nacional de Educação.

Sameroff, A. J., \& Fiese, B. H. (2000). Transactional regulation: The developmental ecology of early interventions. In S. J. Meisels, \& J. P. Shonkoff(Eds.), Handbook of early childhood intervention ( $2^{\mathrm{a}}$ ed., pp. 135-159). Cambridge: Cambridge University Press.

Shonkoff, J. P., \& Phillips, D. A. (2000). From neurons to neighborhoods. The Science of Early Childhood Development. Washington, D.C.: National Academy Press.

United Nations Children's Fund. (2008). A transição dos cuidados na infância: Innocenti Report Card $n .^{\circ} 8$. Florença, Itália: Centro de Pesquisa Innocenti da UNICEF.

van Ijzendoorn, M. H., Tavecchio, L. C., Stams, G. M., Verhoeven, M. E., \& Reiling, E. J. (1998). Quality of center day care and attunement between parents and caregivers: Center day care in cross-national perspective. Journal of Genetic Psychology, 159(4), 437-454. 
Vandell, D., Belsky, J., Burchinal, M., Steinberg, L., Vandergrift, N., $\&$ the NICHD Early Child Care Research Network. (2010). Do effects of early child care extend to age 15 years? Results from the NICHD Study of Early Child Care and Youth Development. Child Development, 81, 737-756.
Vandell, D. L., \& Wolfe, B. (2000). Child care quality: Does it matter and does it need to be improved? Washington, DC: Institute for Research on Poverty, University of WisconsinMadison.

Whitebook, M., Howes, C., \& Phillips, D. (1989). Who cares? Child care teachers and the quality of care in America. Oakland, CA: Child Care Employee Project.

Recebido em 03.07.2013

Primeira decisão editorial em 07.04.2015

Versão final em 05.05.2015

Aceito em 11.06.2015 